\title{
Three-dimensional transoesophageal echocardiographic imaging for support of minimally invasive robotic mitral valve repair using the da Vinci system - first experience in Poland
}

\author{
Katarzyna Kurnicka ${ }^{1}$, Krzysztof Wróbel ${ }^{2}$, Dariusz Zieliński², Zbigniew Juraszyński², Andrzej Biederman², \\ Piotr Pruszczyk ${ }^{1}$ \\ ${ }^{1}$ Department of Internal Medicine and Cardiology, Medical University of Warsaw, Warsaw, Poland \\ ${ }^{2}$ Department of Cardiac Surgery, Medicover Hospital, Warsaw, Poland
}

Adv Interv Cardiol 2019; 15, 3 (57): 382-384

DOI: https://doi.org/10.5114/aic.2019.87902

Minimally invasive robotic mitral valve (MV) repair requires very precise preoperative planning. In order to determine the extent of MV disease, high-quality transthoracic (TTE) followed by transoesophageal (TEE) echocardiography is recommended [1]. Three-dimensional TEE imaging (3D-TEE) significantly complements two-dimensional TEE (2D-TEE) in the operative planning [1, 2].

Two men (45 and 56 years old) with symptomatic severe mitral regurgitation (MR) due to MV prolapse (NYHA II/III) without a history of coronary artery disease were qualified for minimally invasive robotic MV repair using the da Vinci system. The first procedures in Poland were performed in Warsaw in November 2018.

2D-TEE preceding the surgery revealed in both cases a significant posterior leaflet prolapse which resulted in a large MR (wide or eccentric jet, large convergence zone, wide vena contracta) (Figures $1 \mathrm{~A}, \mathrm{C}$ ) without left ventricular dilatation or systolic dysfunction.

As required, a detailed 2D/3D-TEE assessment was made to determine MV topography and the risk of systolic anterior motion. The measurements of each leaflet segment, mitral and tricuspid annular diameter, planar angle between the mitral and aortic annulus and the aortic outflow tract septal thickness and coaptation point to septal distance were taken as recommended [3].

3D-TEE performed in the operating room allowed us to evaluate in detail the mechanism of MR and confirmed at least three broken native chords to the P2 scallop in the first patient (Figure $1 \mathrm{~B}$ ), and P2 prolapse with a significant cleft in the middle of the scallop in the second one (Figure $1 \mathrm{D}$ ). Additionally, the direction of MR jets was mapped and their intensity was assessed by 2D- and 3D-TEE.

TEE was also used to guide a proper placement of two venous cannulas positioned in the superior and inferior venae cavae and an arterial cannula during the institution of cardiopulmonary bypass (CPB). After left atriotomy, the da Vinci camera view revealed the MV anatomy comparable to the view by 3D-TEE (Figure $1 \mathrm{E}$ ).

In the first patient, a triangular resection of the prolapsing part of the leaflet with broken chords was performed. In the second patient, the P2 cleft was closed and two neochords were placed in the lateral and medial papillary muscle and, subsequently, attached to the prolapsing P2 segment. In both patients, a flexible partial band was implanted using interrupted sutures from the medial towards the lateral fibrous triangle (Simulus $35 \mathrm{~mm}$ and Simulus $31 \mathrm{~mm}$, respectively). After CPB was stopped, good MV competence using 2D-TEE and color Doppler was confirmed, and the final result was clearly shown by 3D-TEE (Figure $1 \mathrm{~F}$ ).

It should be emphasized that good quality preoperative TEE imaging plays a pivotal role in MV repair, particularly with the use of the 3D-TEE option [4], which allows one to create a topographic image model of the MV $[2,3]$. Moreover, 3D-TEE echocardiography is a valuable technique for assessment of the MV repair result [4]. This modality also worked well in the first two Polish patients who underwent a robotic MV repair due to severe MR using the da Vinci system.

\section{Conflict of interest}

The authors declare no conflict of interest.

\section{Corresponding author:}

Katarzyna Kurnicka MD, PhD, Department of Internal Medicine and Cardiology, Medical University of Warsaw, 4 Lindleya St, $02-005$ Warsaw, Poland, phone: +48 2250217 96, e-mail: kkurnicka@yahoo.pl

Received: 5.07.2019, accepted: 13.07.2019. 

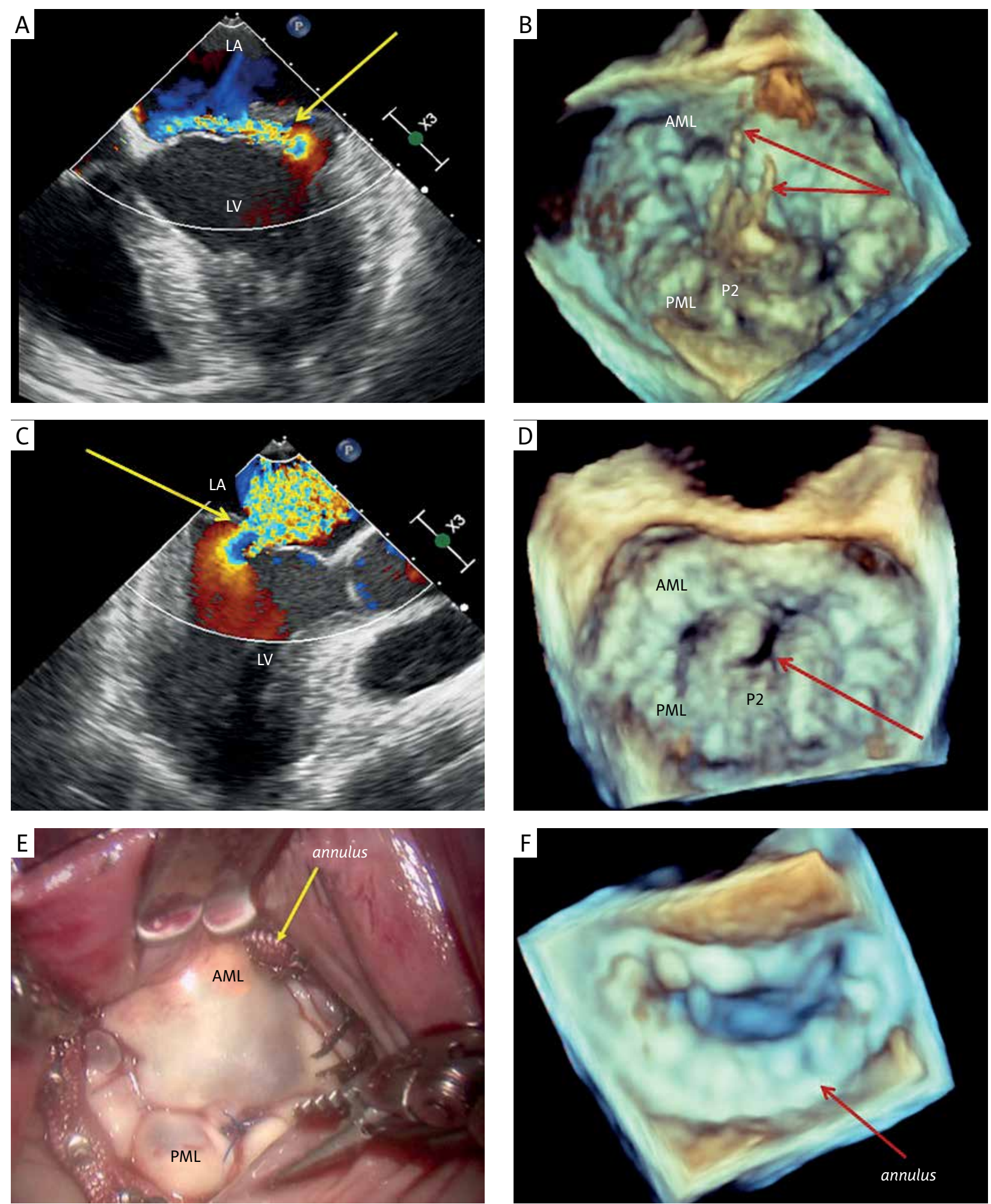

Figure 1. Assessment of the mitral valve prior to and after minimally invasive robotic valve repair using $2 D$ and 3D transoesophageal echocardiography. A - Eccentric jet of significant mitral regurgitation (arrow) - color Doppler. B - "Surgical view" of the mitral valve: posterior leaflet prolapse and broken native chords to P2 scallop (arrows). C - Severe mitral regurgitation: a wide jet and large convergence zone (arrow) - color Doppler. D - "Surgical view" of the mitral valve: posterior leaflet prolapse and a cleft within the P2 segment (arrow). $\mathrm{E}$ - The da Vinci camera view of the mitral valve in the first patient after excision of the prolapsing segment and annulus implantation. $\mathbf{F}-$ Good final result of the robotic procedure assessed by 3D-TEE $A M L$ - anterior mitral leaflet, $L A$ - left atrium, $L V$ - left ventricle, $P M L$ - posterior mitral leaflet, $P 2$ - central segment of posterior mitral leaflet. 


\section{References}

1. Chitwood WR Jr. Robotic mitral valve surgery: overview, methodology, results, and perspective. Ann Cardiothorac Surg 2016; 5: 544-55.

2. Mori M, Yoshimuta T, Ohira M, et al. Impact of real-time three-dimensional transesophageal echocardiography on procedural success for mitral valve repair. J Echocardiogr 2015; 13: 100-6.

3. D'Alonzo RC, Gorrin-Rivas MJ, Mackensen GB. Three dimensional transesophageal echocardiographic planning. In: Atlas of Robotic Cardiac Surgery, Chitwood WR (ed.). Springer, New York 2014; 33-54.

4. Drasutiene A, Aidietiene S, Zakarkaite D. The role of real time three-dimensional transoesophageal echocardiography in acquired mitral valve disease. Semin Cardiovasc Med 2015; 21: 16-26. 